\title{
Caracterización socio-demográfica y clínica de pacientes adultos en ventilación mecánica no invasiva domiciliaria. Ministerio de Salud. Chile
}

\author{
MARIANELA ANDRADE A.*, MÓNICA ANTOLINI T.**, KRISHNNA CANALES H.***, \\ MAURICIO FUENTES A.****, MARICELLA MAZZEI P.***** y CÉSAR MAQUILÓN O.**
}

\section{Socio-demographic and clinical characterization of adult patients in non-invasive home mechanical ventilation. Ministry of Health. Chile}

Introduction: Non-invasive home mechanical ventilation (NIHMV) is delivered in Chile since 2008 throughout a public program, including equipment and professionals. Objectives: 1) Characterize the socio-demographic and clinical profile of the adult patient under NIHMV and 2) Propose improvements in social health care. Methodology: Descriptive cross-sectional study, through face-toface home interview and review of official databases. Sample of 267 subjects, both gender, over 20 years-old, with Chronic Global Respiratory Failure (CGRF) in NIHMV. In 2016 there were 413 active patients. Results: Women 144 (53.9\%), mean age $58.6 \pm 18$ years-old; $25.5 \%$ had COPD and 24\% had a hypoventilation obesity syndrome, average $\mathrm{PaCO}_{2}$ at the time of admission to program was $59 \pm 11$ $\mathrm{mmHg}$, they were ventilated since $3.2 \pm 2.4$ years, $7.6 \pm 2.4 \mathrm{~h}$ a day. Subjects "without instruction" and with "incomplete basic education" represents $40.7 \%$ of the sample. $46.4 \%$ were retired persons; $3 \%$ lived in a precarious hut; $19.8 \%$ cohabited with relatives or close friends; $49 \%$ did not have a partner; 4.8\% lived alone; $68.6 \%$ was oxygen dependent; $40 \%$ had an altered Golberg test; $17.7 \%$ "has a major limitation, unable to perform self-care". Conclusions: Our patients have a severe socio-demographic and clinical deterioration, due to low schooling level, predominance of older adults, a major incapacity for working and patients have a more severe disease with higher baseline $\mathrm{PaCO}_{2}$ levels, compared to European studies. NIHMV programs must adopt a social health approach and be inserted into the health network in social service, and cardiovascular and mental health programs.

Key words: Non-invasive home mechanical ventilation; Obesity Hypoventilation Syndrome; Pulmonary Disease, Chronic Obstructive; Cross sectional studies; Adult; Humans.

\section{Resumen}

Introducción: La ventilación mecánica no invasiva domiciliaria (VMNID) se entrega en Chile desde el año 2008 mediante un programa público. Incluye equipamiento y profesionales. Objetivos: 1) Caracterizar el perfil socio-demográfico y clínico del usuario adulto con VMNID y 2) Proponer mejoras

Este trabajo no contó con ningún financiamiento de institución gubernamental, sociedad médica, empresa u otra organización.

\footnotetext{
* $\quad$ Kinesióloga, Programa AVNIA (Asistencia Ventilatoria no Invasiva en Adultos AVNIA), MINSAL (Ministerio de Salud) y Hospital San José SSMN (Servicio de Salud Metropolitano Norte). Santiago. Magíster de Salud Pública, Escuela de Salud Pública, Universidad de Chile. Santiago, Chile.

** Broncopulmonar, Programa AVNIA, MINSAL y Departamento de Enfermedades Respiratorias, Clínica Dávila, Santiago, Chile.

*** Enfermera, Programa AVNIA, MINSAL y enfermera supervisora de la Unidad de Cuidados Intensivos, Clínica Dávila, Santiago, Chile.

**** Estadístico, Escuela Salud Pública, Universidad de Chile. Santiago, Chile.

*****Socióloga, Escuela Salud Pública, Universidad de Chile. Santiago, Chile.
} 
de atención socio-sanitaria. Método. Estudio descriptivo transversal, mediante entrevista presencial domiciliaria y revisión de bases de datos oficiales. Muestra de 267 sujetos, ambos géneros, mayores de 20 años, con Falla Respiratoria Global Crónica (FRGC) en VMNID. En 2016 habia 413 pacientes activos. Resultados: Mujeres 144 (53,9\%), edad media 58,6 \pm 18 años. 25,5\% tienen EPOC y 24\% síndrome hipoventilación obesidad, la $\mathrm{PaCO}_{2}$ promedio de ingreso al programa fue de $59 \pm 11 \mathrm{mmHg}$. Ventilados desde 3,2 \pm 2,4 años, por 7,6 2,4 h/dia. Sujetos "sin instrucción" y con "educación básica incompleta" representan el 40,7\% de la muestra. 46,4\% eran jubilados, 3\% vive en mediaguas, $19,8 \%$ reside como allegado, 49\% no contaba con pareja, el 4,8\% vivía solo, 68, $6 \%$ eran dependientes de oxígeno. Test de Golberg estuvo alterado en un 40\%. 17,7\% "posee mayor limitación, incapaz de realizar el autocuidado". Conclusiones: Nuestros pacientes tienen un deterioro socio-demográfico y clínico severo, por baja escolaridad, predominio de adultos mayores, mayor incapacidad laboral, son enfermos más graves con niveles basales de $\mathrm{PaCO}_{2}$ más altos, en comparación a estudios europeos. Los programas de VMNID deben adoptar un enfoque socio-sanitario y estar insertos en la red de salud tanto en servicio social, salud cardiovascular y mental.

Palabras clave: Ventilación mecánica no invasiva domiciliaria; Síndrome hipoventilación obesidad; Enfermedad pulmonar obstructiva crónica; Estudios de corte transversal; Adulto; Humanos.

\section{Introducción}

El uso de la ventilación mecánica no invasiva domiciliaria (VMNID) en sujetos con falla respiratoria global crónica (FRGC) ha aumentado por la mayor expectativa de vida, la profundización del envejecimiento y la mayor prevalencia de enfermedades pulmonares crónicas, esto asociado al rápido desarrollo tecnológico biomédico ha permitido que los dispositivos requeridos (ventiladores mecánicos, generadores de flujo) sean menos costosos y más portables ${ }^{1}$. La FRGC puede ser de origen pulmonar o extra pulmonar, y quienes la padecen presentan una mayor tasa de morbimortalidad y requieren recursos sanitarios costosos para el sistema, tales como: ocupación de camas críticas, atenciones en servicios de urgencia y consulta de especialidad, siendo esto de mayor importancia en el período de alta circulación viral (invierno) ${ }^{2}$. No se disponen de cifras que estimen la prevalencia de la VMNID en Chile ni en América Latina. En Europa, el estudio EUROVENT estimó en el año 2005, una prevalencia media de ventilación mecánica invasiva y no invasiva de 6,6 por 100.000 habitantes $^{3}$. En Chile, el año 2008 el Ministerio de Salud (MINSAL), implementó en la red pública el programa AVNIA (Asistencia Ventilatoria no Invasiva en Adultos), como una estrategia para optimizar los recursos de salud, realizando un seguimiento médico protocolizado en el hospital base del paciente, implementando un perfil de atención más humanizado. Este programa se estableció con los siguientes objetivos: 1) Ventilar a adultos con hipoventilación alveolar crónica; 2) Transferir tecnología al domicilio, otorgando capacitación a familiares y supervisión domici- liaria por profesionales entrenados; 3) Reducir los días de hospitalización por año y 4) Mejorar la calidad de vida ${ }^{4}$. Al año 2016 habían ingresado al programa 780 pacientes adultos y permanecían activos 413 mayores de 20 años de edad, portadores de FRGC, identificándose como las más prevalentes a la enfermedad pulmonar obstructiva crónica (EPOC) y al Síndrome Hipoventilación Obesidad (SHO), ambos de especial importancia para la salud pública, ya que se catalogan como enfermedades evitables, relacionadas a la alta prevalencia del tabaquismo y de la obesidad ${ }^{2,5}$. Se realizó la primera caracterización de una muestra de adultos en VMNID en Chile. El programa AVNIA beneficia a pacientes del seguro de salud estatal (FONASA) el cual cubre alrededor del $70 \%$ de la población y representa al grupo de personas con el nivel de ingresos monetarios más bajos del país, es por ello relevante conocer el perfil del usuario ventilado en domicilio. Se plantearon los siguientes objetivos: 1) Caracterizar el perfil socio-demográfico y clínico del usuario adulto de VMNID; 2) Describir parámetros ventilatorios y de adherencia a este tipo de soporte y 3) Proponer mejoras a la atención del usuario con enfoque socio-sanitario en base a los hallazgos del estudio.

\section{Pacientes y Método}

Es un estudio descriptivo y transversal con un muestreo no probabilístico por conveniencia. La fuente de sujetos para la muestra fue el programa AVNIA, MINSAL, el cual a la fecha del estudio (año 2016) contaba con 413 pacientes activos (en cinco regiones del país: V, VI, VII, VIII y Metro- 
politana). Se incluyeron sujetos de ambos sexos, mayores de 20 años de edad, con diagnóstico de FRGC según normativa técnica MINSAL ${ }^{4}$ confirmada por 12 neumólogos AVNIA de los hospitales base correspondientes. Los pacientes debían estar utilizando equipos de presión positiva binivelada por lo menos 30 días continuos, desde su ingreso al programa. Se excluyó a los sujetos que no desearon participar en el estudio, a quienes padecieran deterioro cognitivo o neurológico que impidiera la comprensión de las instrucciones (lo cual es un aspecto clave para responder preguntas de auto reporte), a los que estuvieran cursando con una exacerbación o descompensación cardiorrespiratoria relevante documentada y a quienes hubieran cambiado a ventilación invasiva domiciliaria. Se estableció una clasificación de ocho grupos diagnósticos según las enfermedades de base que generaran la FRGC, siendo las más frecuentes en el programa de VMNID nacional y en los referentes de Europa ${ }^{7-9}$, los siguientes grupos: 1) EPOC; 2) EPOC asociada a síndrome de apnea obstructiva de sueño (Síndrome de sobre posición EPOC-SAOS); 3) Secuela de tuberculosis; 4) Bronquiectasias no fibrosis quística; 5) Enfermedades neuromusculares; 6) Cifoscoliosis; 7) Síndrome Hipoventilación Obesidad (SHO) y 8) Misceláneas.

El protocolo fue ejecutado en el domicilio por enfermeros y kinesiólogos del programa, registrándose para cada sujeto una hoja de antecedentes socio-demográficos, clínicos y ventilatorios elaborada para el estudio y, además, se revisó el equipamiento en uso. Algunas de las variables clínicas registradas fueron: tabaquismo, uso de oxigenoterapia, comorbilidades, nivel de actividad física (mediante la pauta 'NIV Audit' de la British Thoracic Society (BTS), Standards of Care Committee in Non Invasive Ventilation ${ }^{11}$ y entre las variables ventilatorias registradas se incluyó: modo ventilatorio, presiones inspiratoria y espiratoria positivas de vía aérea (IPAP, EPAP), frecuencia respiratoria de respaldo, máscara utilizada, horas de uso del ventilador en los últimos siete días, patrón de uso y tolerancia ${ }^{12,13}$. Después de la recolección de datos en el domicilio, la variable "diagnóstico" fue verificada en bases de datos MINSAL por los autores y por medio del número de cédula de identidad se verificaron las comorbilidades GES (garantías explicitas en salud) ${ }^{14,15}$. Se describen los valores basales de gases arteriales ${ }^{17}$ y espirometría. Los espirómetros utilizados fueron MGC Diagnostics (Medical Graphic) modelo "CPFS/D USB Spirometer" software "Breeze Suite" los cuales siguieron protocolo de calibración según manual de procedimientos SER y fueron ejecutadas por el profesional a cargo del laboratorio de función pulmonar de los hospitales base correspondiente a la muestra.

En cuanto al seguro de salud público (FONASA) se utilizó la información del nivel de ingreso, el cual se divide en 4 tramos: A, B, C y $\mathrm{D}$ (siendo " $\mathrm{A}$ " el tramo para sujetos carentes de recursos económicos, no cotizantes, con pensiones de gracia o asistenciales, hasta el tramo " $D$ " constituido por sujetos con ingresos mensuales mayores a $\$ 385.441$, equivalente a 610 USD aproximadamente) ${ }^{16}$.

El estudio contó con la aprobación del Comité de Ética del Servicio de Salud Metropolitano Norte. Todos los sujetos que aceptaron participar firmaron un consentimiento informado.

El análisis estadístico se realizó con estadística descriptiva mediante frecuencias absolutas y porcentajes para variables cualitativas, medidas de tendencia central y dispersión para variables cuantitativas. Se utilizó el programa estadístico SPSS (Statistical Package for the Social Sciences) versión 21.

\section{Resultados}

Todos los pacientes $(n=267)$ cumplieron los criterios de inclusión. Sus principales características se muestran en las Tablas 1 y 2. La totalidad de ellos se encontraba utilizando VMNID con equipos y accesorios adquiridos por licitaciones públicas ministeriales que se realizan en base a especificaciones técnicas. Un 74,2\% era de la Región Metropolitana y un 25,8\% de otras regiones (V, VI, VII y VIII), esta distribución se debe a la mayor densidad poblacional en la capital del país.

La edad de los participantes fluctuó entre los 20 y 88 años, con una mediana de 63 años (p25 = 51 años y p75 = 72 años), predominando los adultos mayores (Figura 1). El 41,2\% de los pacientes son sujetos con educación "básica incompleta" y "sin instrucción". Para ambos grupos las mujeres predominaron con el menor nivel educacional. El 47,6\% se encontraba viviendo en pareja. Al consultar sobre el número de personas que habitan en la vivienda, el 71,2\% se concentró en hogares donde residen " 2 a 5 personas", $\sin$ embargo, el 4,9\% "vive solo" (ocho de ellos son dependientes del oxígeno).

Los "jubilados" (por invalidez y vejez) representaron el 47,5\% de la muestra (Tabla 1), seguidos por "dueñas/os de casa" en un 29,6\% ( $\mathrm{n}=70$ mujeres y 9 hombres). Los "estudiantes" correspondieron al 6,4\% y con edades entre 20 a 
Tabla 1. Características socio-demográficas de los 267 pacientes con ventilación mecánica no invasiva domiciliaria-VMNID

\begin{tabular}{|c|c|c|c|}
\hline Mujeres & $\mathrm{n}(\%)$ & 144 & $(53,9)$ \\
\hline Edad, en años & Mediana $(\mathrm{p} 25-\mathrm{p} 75)^{*}$ & 63( & $(51-72)$ \\
\hline \multirow{6}{*}{\multicolumn{2}{|c|}{$\begin{array}{l}\text { Nivel educacional } \\
\text { Sin instrucción/Básica incompleta } \\
\text { Básica completa } \\
\text { Media incompleta } \\
\text { Media incompleta/Superior incompleta } \\
\text { Superior completa }(* *)\end{array}$}} & $\mathrm{n}$ & $(\%)$ \\
\hline & & 10 & $(41,2)$ \\
\hline & & 38 & $(14,2)$ \\
\hline & & 34 & $(12,7)$ \\
\hline & & 66 & $(24,7)$ \\
\hline & & 19 & $(7,1)$ \\
\hline \multicolumn{2}{|c|}{ Estado civil } & $\mathrm{n}$ & $(\%)$ \\
\hline \multicolumn{2}{|l|}{ Casado (a) } & 119 & $(44,6)$ \\
\hline \multicolumn{2}{|c|}{ Conviviente } & 8 & $(3,0)$ \\
\hline \multicolumn{2}{|c|}{ Soltero/a $(* * *)$} & 84 & $(31,5)$ \\
\hline \multicolumn{2}{|c|}{ Viudo (a) $(* * *)$} & 42 & $(15,7)$ \\
\hline \multicolumn{2}{|c|}{ Situación laboral } & $\mathrm{n}$ & $(\%)$ \\
\hline \multicolumn{2}{|c|}{ Dueño (a) de casa } & 79 & $(29,6)$ \\
\hline \multicolumn{2}{|c|}{ Estudiante } & 17 & $(6,4)$ \\
\hline \multicolumn{2}{|c|}{ Jubilado (a) / Pensión de vejez } & 66 & $(24,7)$ \\
\hline \multicolumn{2}{|c|}{ Trabajo estable } & 25 & $(9,4)$ \\
\hline \multicolumn{2}{|c|}{ Trabajo ocasional } & 11 & $(4,1)$ \\
\hline \multicolumn{2}{|c|}{ Cesante } & 8 & $(3,0)$ \\
\hline \multicolumn{2}{|c|}{$\begin{array}{l}\text { Ingresos según Seguro de Salud } \\
\text { (FONASA) }\end{array}$} & $\mathrm{n}$ & $(\%)$ \\
\hline \multicolumn{2}{|c|}{$\begin{array}{l}\text { Tramo A (carente de recursos, } \\
\text { pensión de gracia) }\end{array}$} & 20 & $(7,5)$ \\
\hline \multicolumn{2}{|c|}{ Tramo B $(<\$ 264.000)$} & 226 & $(84,6)$ \\
\hline \multicolumn{2}{|c|}{ Tramo C (\$264.001 - \$385.440) } & 1 & $(0,4)$ \\
\hline \multicolumn{2}{|c|}{ Tramo D $(>\$ 385.441)$} & 10 & $(3,7)$ \\
\hline \multicolumn{2}{|c|}{$\begin{array}{l}\text { Bloqueado (a) en el sistema } \\
\text { (sin cobertura) }\end{array}$} & 10 & $(3,7)$ \\
\hline \multicolumn{2}{|c|}{ Tipo de vivienda } & $\mathrm{n}$ & $(\%)$ \\
\hline \multicolumn{2}{|c|}{$\begin{array}{l}\text { Material sólido (casa o departamento } \\
\text { en edificio) }\end{array}$} & 185 & $(69,3)$ \\
\hline \multicolumn{2}{|c|}{ Mixto (madera y cemento) } & 73 & $(27,3)$ \\
\hline \multicolumn{2}{|c|}{ Madera } & 3 & $(1,1)$ \\
\hline \multicolumn{2}{|c|}{$\begin{array}{l}\text { Mediagua (vivienda de materiales } \\
\text { prefabricados) }\end{array}$} & 6 & $(2,2)$ \\
\hline \multicolumn{2}{|c|}{ Calidad en que reside } & $\mathrm{n}$ & $(\%)$ \\
\hline \multicolumn{2}{|c|}{ Propietario (a) } & 190 & $(71,2)$ \\
\hline Arrendatario & & 21 & $(7,9)$ \\
\hline Allegado (a) & & 55 & $(20,6)$ \\
\hline Asentamient & formal (campamento) & 1 & $(0,4)$ \\
\hline $\begin{array}{l}\text { Número de pe } \\
\text { vivienda }\end{array}$ & has que habitan en la & $\mathrm{n}$ & $(\%)$ \\
\hline 1 persona $(\mathrm{v}$ & solo/a) & 13 & $(4,9)$ \\
\hline 2 a 5 person & & 190 & $(71,2)$ \\
\hline 6 a 9 person & & 57 & $(21,3)$ \\
\hline 10 a 14 pers & & 6 & $(2,2)$ \\
\hline Más de $15 \mathrm{p}$ & & 1 & $(0,4)$ \\
\hline
\end{tabular}

*p25= percentil 25; p75= percentil 75. **Educación Superior incluye Técnica y Universitaria. ***En la totalidad de estos casos, declaran estar sin pareja al momento de la encuesta.
Tabla 2. Características clínicas de los 267 pacientes con ventilación mecánica no invasiva domiciliaria-VMNID

\begin{tabular}{|c|c|}
\hline $\begin{array}{l}\text { Diagnóstico que causa la hipoventilación } \\
\text { (Grupos) }\end{array}$ & $\mathrm{n} \quad(\%)$ \\
\hline 1) $\mathrm{EPOC}$ & $68(25,5)$ \\
\hline 2) EPOC-SAOS & $39(14,6)$ \\
\hline 3) Secuelas de tuberculosis & $9 \quad(3,4)$ \\
\hline 4) Bronquiectasias no fibrosis quística & $19 \quad(7,1)$ \\
\hline 5) Neuromuscular & $34(12,7)$ \\
\hline 6) Cifoscoliosis & $27(10,1)$ \\
\hline 7) $\mathrm{SHO}$ & $64(24,0)$ \\
\hline 8) Diagnósticos misceláneos & $7 \quad(2,6)$ \\
\hline $\begin{array}{l}\text { Tiempo en Programa de VMNID, en años } \\
\text { Media } \pm \text { DS }\end{array}$ & $3,2 \pm 2,4$ \\
\hline $\begin{array}{l}\text { Uso de ventilación mecánica, en h/día } \\
\text { Media } \pm \text { DS }\end{array}$ & $7,6 \pm 2,3$ \\
\hline $\begin{array}{l}\text { Parámetros ventilatorios } \\
\text { IPAP }\left(\mathrm{cm} \mathrm{H} \mathrm{H}_{2} \mathrm{O}\right) \\
\operatorname{EPAP}\left(\mathrm{cm} \mathrm{H}_{2} \mathrm{O}\right)\end{array}$ & $\begin{array}{r}\text { Media } \pm \text { DS } \\
18,7 \pm 3,5 \\
8,2 \pm 1,7\end{array}$ \\
\hline Modalidad ventilatoria & $\mathrm{n} \quad(\%)$ \\
\hline Espontáneo (S) & $12 \quad(4,5)$ \\
\hline Espontáneo / temporizado (S/T) & $221(82,8)$ \\
\hline $\begin{array}{l}\text { Espontáneo / tiempo más volumen } \\
\text { asegurado (híbrido) }\end{array}$ & $34(12,7)$ \\
\hline Función respiratoria (espirometría) & $\mathrm{n} \quad(\%)$ \\
\hline Normal & $2(0,7)$ \\
\hline Obstructiva & $138 \quad(51)$ \\
\hline Restrictiva & $127(47,6)$ \\
\hline Oxígeno dependiente & $\mathrm{n} \quad(\%)$ \\
\hline Sí, usuarios 24 h/día & $91(34,1)$ \\
\hline Sí, usuarios $>15$ h/día & $89(33,3)$ \\
\hline No & $87(32,6)$ \\
\hline Tabaquismo & $\mathrm{n} \quad(\%)$ \\
\hline Sí, activo & $6 \quad(2,2)$ \\
\hline Sí, detenido & $161(60,3)$ \\
\hline Nunca & $100(37,5)$ \\
\hline Comorbilidad cardiovascular & $\mathrm{n} \quad(\%)$ \\
\hline Hipertensión arterial & $149(60,1)$ \\
\hline Hipertensión arterial asociada a diabetes & $67(27,0)$ \\
\hline Nivel de funcionalidad (NIV Audit BTS) & $\mathrm{n} \quad(\%)$ \\
\hline Actividad normal sin restricción & $30(11,2)$ \\
\hline $\begin{array}{l}\text { Actividad fuerte limitada, capaz de } \\
\text { trabajos ligeros }\end{array}$ & $89(33,3)$ \\
\hline $\begin{array}{l}\text { Actividad habitual muy limitada, capaz } \\
\text { de autocuidados }\end{array}$ & $98(36,7)$ \\
\hline $\begin{array}{l}\text { Actividad habitual muy limitada, } \\
\text { incapaz de autocuidados }\end{array}$ & $27(10,1)$ \\
\hline $\begin{array}{l}\text { Confinado a cama/sillón, incapaz de } \\
\text { autocuidados }\end{array}$ & $(8,6)$ \\
\hline
\end{tabular}

$\mathrm{SHO}=$ síndrome obesidad-hipoventilación. $\mathrm{DS}=$ Desviación estándar. IPAP $=$ Presión positiva inspiratoria. $\mathrm{EPAP}=$ Presión positiva espiratoria. $\mathrm{NIV}=$ Non Invasive Ventilation. 


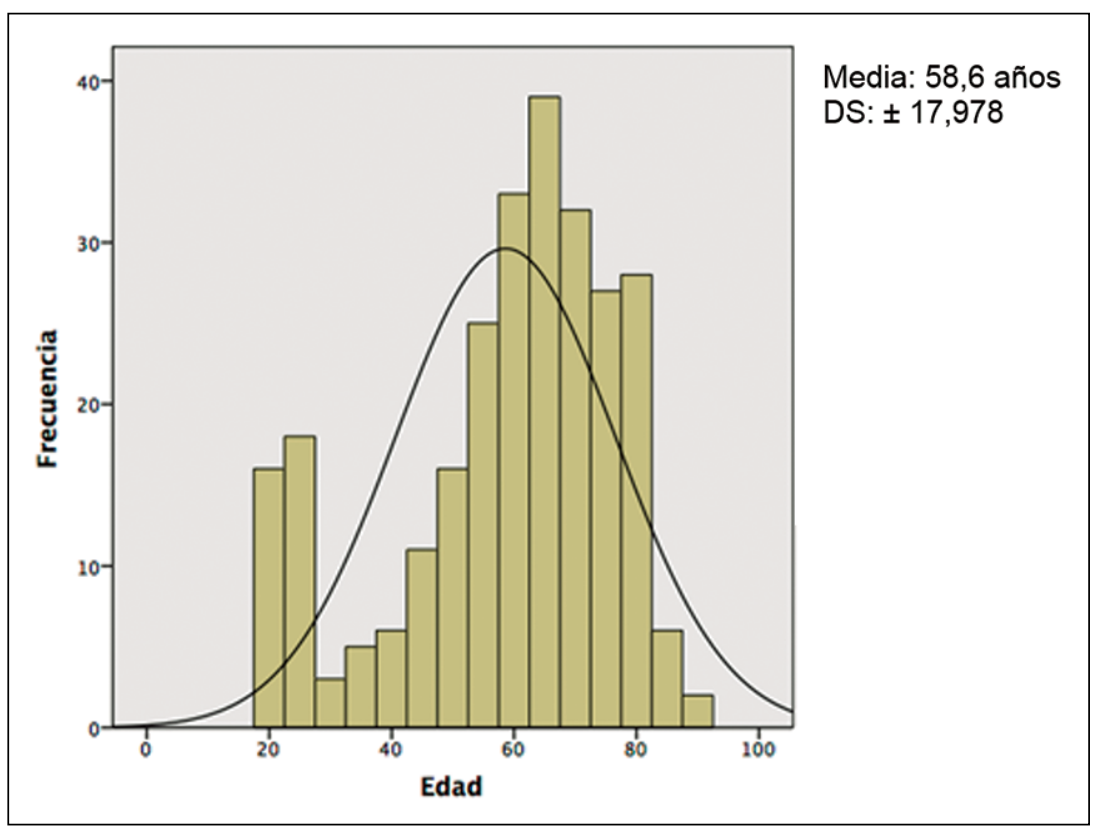

Figura 1. Gráfico de distribución por edad de los 267 pacientes en ventilación mecánica no invasiva domiciliaria. Edad: expresada en años. Frecuencia: número de pacientes por tramo de edad.

25 años. Sólo 36 pacientes estaban laboralmente activos (30 hombres) cuyos diagnósticos fueron Cifoscoliosis y SHO, cuya edad promedio fue de $50,7 \pm 14,1$ años. Declararon desempeñar oficios con baja carga física, tales como: conserje de edificio, comercio informal, chofer de vehículo menor y labores administrativas (uso de computador). Respecto de los "cesantes", su mediana de edad es de 43,5 años (p25 = 28,5 y p75 $=49,2$ años) y sus diagnósticos fueron bronquiectasias, enfermedades neuromusculares, cifoscoliosis y SHO. Según el seguro de salud ${ }^{16}$ el 7,5\% pertenecía al Fondo Nacional de Salud (FONASA) tramo A (carente de recursos, condición de indigencia, pensión asistencial), el 84,6\% a tramo B (ingreso menor a $\$ 264.000$ mensual equivalente a 420 USD) y el 3,7\% tenía su previsión inactiva ("bloqueada"), estos últimos pacientes eran cesantes menores de 60 años (Tabla 1).

Respecto de las características clínicas (Tabla 2), los diagnósticos predominantes fueron EPOC con un $25,5 \%$, SHO con un $24,0 \%$ y síndrome de sobre posición EPOC-SAOS en un 14,6\%. El grupo diagnóstico con los sujetos de mayor edad fue la EPOC con una mediana de 71 años (p25 = 64 y p75 $=75$ años), mientras, el grupo de menor edad fueron los neuromusculares con una mediana de 23,5 años. Los diagnósticos de

Tabla 3. Distribución por diagnóstico y sexo del grupo de enfermedades neuromusculares en ventilación mecánica no invasiva domiciliaria-VMNID

\begin{tabular}{|lccc|}
\hline Enfermedad neuromuscular & Hombre & Mujer & Total \\
\hline Distrofia muscular de Duchenne & 12 & 0 & 12 \\
Enfermedad de Steinert & 6 & 0 & 6 \\
\hline Atrofia muscular espinal tipo II & 1 & 3 & 4 \\
Miopatía congénita & 3 & 1 & 4 \\
Parálisis diafragmática & 1 & 3 & 4 \\
Esclerosis lateral amiotrófica & 0 & 1 & 1 \\
Distrofia muscular de Ulrich & 1 & 0 & 1 \\
Polineuropatía sensorio motriz & 0 & 1 & 1 \\
Enfermedad de Pompe & 0 & 1 & 1 \\
Total & 24 & 10 & 34 \\
\hline
\end{tabular}


los portadores de enfermedades neuromusculares que debutaron en la adultez correspondieron a parálisis diafragmática y esclerosis lateral amiotrófica (ELA). Las enfermedades clasificadas como Misceláneas en conjunto representaron el 2,6\% de la muestra, con predominio de mujeres y una mediana de edad de 59 años (Tabla 4).

Los 267 pacientes tenían una $\mathrm{PaCO}_{2}$ y un $\mathrm{pH}$ en sangre arterial promedio \pm DS de $59 \pm 11$ mmHg y 7,36 $\pm 0,04$, respectivamente (Tabla 5) y presentaron una permanencia promedio en VMNID de 3,2 $\pm 2,4$ años, el promedio del uso diario del ventilador fue de 7,6 $\pm 2,4 \mathrm{~h}$ (calculado en base al uso efectuado los siete días previos a la visita) (Tabla 2). Los ocho grupos diagnósticos estudiados mostraron un cumplimiento de horas de uso de ventilador sobre lo recomendado (mayor a 4 h). El patrón de uso del ventilador fue de preferencia "nocturno exclusivo", es decir, el $78,3 \%$ de los participantes utiliza el ventilador mientras duerme y en los casos de insuficiencia respiratoria más avanzada el patrón de uso es "nocturno más horas de apoyo diurno". El 71,2\%, usó máscara oronasal y la tolerancia a la venti- lación fue reportada como buena en un 91,4\%. Se reportaron 15 casos $(5,6 \%)$ que desarrollaron úlceras por presión facial (UPP) a nivel del dorso de la nariz y $19(7,1 \%)$ con conjuntivitis y dermatitis facial. El 68,5\% de los pacientes también pertenecen a un programa de oxigenoterapia domiciliaria, conectando la fuente de oxígeno al ventilador mecánico. El nivel de funcionalidad general de la pauta 'NIV Audit' (BTS)" mostró que el $18,7 \%$ posee "mayor limitación y es incapaz de realizar el autocuidado". En este grupo destacan los pacientes con enfermedad neuromuscular.

El 60,3\% indicó "tabaquismo detenido" (seis meses previos a la encuesta), el 37,5\% refiere "nunca haber fumado" (principalmente enfermos neuromusculares) y el 2,2\% declara "tabaquismo activo" (Tabla 2). Los fumadores activos ( $\mathrm{n}=6$, mujeres $=3$ ), tenían una mediana de edad de 64,6 años, pertenecían a diferentes grupos diagnósticos y niveles educacionales, vivían en pareja y eran capaces de realizar su autocuidado.

Las comorbilidades más significativas, revisadas en el Sistema de Gestión de Garantías Explí-

Tabla 4. Distribución por diagnósticos de las enfermedades incluidas en 'Grupo misceláneo' de pacientes en ventilación mecánica no invasiva domiciliaria-VMNID

\begin{tabular}{|lccc|}
\hline Diagnóstico & Hombre & Mujer & Total \\
\hline Asma bronquial de difícil control & 0 & 2 & 2 \\
\hline Bronquiolitis obliterante postrasplante pulmonar & 1 & 0 & 1 \\
\hline Síndrome de Ondina & 0 & 1 & 1 \\
Síndrome de Pfeiffer & 0 & 1 & 1 \\
\hline Secuela de toracoplastia, neumonectomía & 0 & 2 & 2 \\
Total & 1 & 6 & 7 \\
\hline
\end{tabular}

Tabla 5. Valores promedio basales de $\mathrm{pH}$ y $\mathrm{PaCO}_{2}$ según grupo diagnóstico en los 267 pacientes en ventilación mecánica no invasiva domiciliaria-VMNID

\begin{tabular}{|lccc|}
\hline Grupo diagnóstico & n pacientes & $\mathbf{p H}$ & $\mathbf{P a C O}_{\mathbf{2}}(\mathbf{m m H g})$ \\
\hline EPOC & 68 & $7,36 \pm 0,04$ & $59,8 \pm 9,42$ \\
\hline EPOC + SAHOS & 39 & $7,36 \pm 0,04$ & $61,7 \pm 11,6$ \\
\hline Secuelas de tuberculosis & 9 & $7,35 \pm 0,03$ & $60,2 \pm 6,26$ \\
Bronquiectasias no FQ & 19 & $7,38 \pm 0,03$ & $59,5 \pm 5,39$ \\
Neuromusculares & 34 & $7,38 \pm 0,03$ & $57 \pm 23,3$ \\
Cifoscoliosis & 27 & $7,36 \pm 0,04$ & $57,3 \pm 9,7$ \\
Síndrome hipoventilación obesidad & 64 & $7,37 \pm 0,05$ & $58,9 \pm 10,5$ \\
Grupo misceláneo & 7 & $7,37 \pm 0,01$ & $59,3 \pm 13,9$ \\
Total & 267 & $7,36 \pm 0,04$ & $59 \pm 11$ \\
\hline
\end{tabular}

Los valores de $\mathrm{pH}$ y $\mathrm{PCO}_{2}$ en sangre arterial se expresan en promedio \pm DS. FQ: fibrosis quística. 
citas en Salud (SIGGES) FONASA, mostraron prevalencias de hipertensión arterial (HTA) de $60,1 \%$, diabetes mellitus (DM) 30,8\%, HTA asociada a DM $27 \%$ (Tabla 2), accidente cerebrovascular $(\mathrm{ACV}) 3,4 \%$, insuficiencia renal crónica terminal (IRCT) $4,5 \%$, cataratas en un $16 \%$, cáncer de diversos orígenes $5,2 \%$, hipotiroidismo $7,5 \%$ y requerimiento de órtesis (ayuda técnica para limitación funcional, tales como: silla de ruedas, bastón, carro andador) en el $23,6 \%$ de los sujetos (Tabla 2).

\section{Discusión}

El propósito de este estudio fue obtener información relevante para los clínicos que manejan pacientes en VMNID y los que implementan y/o evalúan programas que imparten esta prestación de salud. Esta investigación, es la primera evaluación socio-demográfica de adultos con FRGC de Chile, además de contar con diagnósticos realizados por neumólogos. Las evaluaciones fueron realizadas en forma presencial (domiciliaria), incluyendo sujetos de cinco regiones del país, tanto de sectores urbanos como rurales enriqueciendo la diversidad socio-cultural de la muestra.

Comparado con estudios similares, este contó con un número mayor de participantes que trabajos europeos, como un estudio alemán ${ }^{7}$ que contaba con una muestra de 226 y el de España con 115 pacientes $^{8}$, además que no consideraban detallar el aspecto socio-demográfico. Una limitación para lograr una muestra mayor fue principalmente el deterioro cognitivo $\mathrm{y} / \mathrm{o}$ del estado de salud de algunos pacientes, además que estructuralmente la población estaba acotada.

En esta muestra destacó la vulnerabilidad social de un alto porcentaje de los sujetos, que se expresó en su baja escolaridad, predominio de adultos mayores y mayor situación de incapacidad laboral, parámetros que mostraron deterioro más profundo respecto del grupo VMNID investigado en España ${ }^{8}$. Si bien, los reportes de los pacientes en VMNID de Alemania, España e Inglaterra muestran población envejecida, esta corresponde a sujetos con mayores niveles de escolaridad, menor vulnerabilidad social y menor grado de severidad clínica de la FRGC comparada con los pacientes de nuestro estudio. El nivel de hipercapnia promedio que se ha reportado en los usuarios del programa AVNIA en Chile (Tabla $5)^{17}$ es mayor en todos los grupos diagnósticos, comparado con lo que reporta Alemania ${ }^{7,18}$ y España $^{8}$ en sus muestras de pacientes ventilados. En Chile, los enfermos tienen promedios basales más altos de $\mathrm{PaCO}_{2}(\mathrm{mmHg})$. En efecto nuestros pacientes con EPOC tienen una $\mathrm{PaCO}_{2}$ promedio de $59,8 \mathrm{mmHg}$ mientras que en Alemania tienen 45,8 y España $52 \mathrm{mmHg}$. Nuestros pacientes EPOC-SAHOS tienen $\mathrm{PaCO}_{2}$ promedio de 61,7 $\mathrm{mmHg}$ y las enfermedades misceláneas 59,3 $\mathrm{mmHg}$; mientras que estas series estudiadas en Alemania y España no tienen datos disponibles.

En Chile, los pacientes con diagnóstico de $\mathrm{SHO}$ tienen en promedio $\mathrm{PaCO}_{2}$ de $58,9 \mathrm{mmHg}$, la serie de España $45 \mathrm{mmHg}$ y la de Alemania no lo tiene disponible, finalmente en nuestro grupo los enfermos Neuromusculares (Tablas 3 y 5) tienen $\mathrm{PaCO}_{2}$ en promedio de $57 \mathrm{mmHg}$, Alemania 42,6 y España 43,6 mmHg. Esta diferencia en los niveles de $\mathrm{PaCO}_{2}$ se puede atribuir a los siguientes factores: mayor severidad de la enfermedad de base al momento de ingreso al programa, disponibilidad limitada de ventiladores para asegurar la cobertura oportuna de todos los pacientes que cumplen criterios de ingreso, escaso conocimiento de otros especialistas, sobre la disponibilidad y uso precoz de la ventilación domiciliaria. En Francia la federación ANTADIR ${ }^{10}$ tiene una experiencia de más de 30 años en VMNID con más de 175.000 ingresados con un manejo integral (este número incluye un número significativo de pacientes con SAOS, pero sin FRGC). En Chile a diferencia de Francia, se tiene una experiencia menor a 10 años en ventilación domiciliaria adulto con prestaciones acotadas y que requiere el apoyo de la atención primaria. Habitualmente los programas nuevos tienden a incluir primero a pacientes más severos y a medida que progresan en el tiempo ingresan con más precocidad pacientes de menor severidad dependiendo de sus recursos (cupos).

Para este estudio, destaca una buena tolerancia general a la VMNID por parte de los usuarios y buena adherencia a la terapia, además de un bajo reporte de efectos adversos.

En el ámbito de las comorbilidades (Tabla 2), la cardiovascular fue la más significativa. El 60,1\% padecía hipertensión arterial (HTA), duplicando la prevalencia nacional $(26 \%)^{5}$. La HTA asociada a diabetes mellitus (DM) fue de $27 \%$. Por esto, se recomienda que los profesionales que trabajan en programas de VMNID, eduquen y supervisen a los pacientes en el uso efectivo de la terapia cardiovascular como parte del protocolo de atención integral. El tabaquismo en esta muestra alcanzó un $62,5 \%$, superando la prevalencia de la población adulta, la cual es de 40,6\% según la Encuesta Nacional de Salud 5 . El 2,2\% de los pacientes a pesar de su gravedad y pronóstico declaró "tabaquismo activo". No se hallaron re- 
portes que describan el hábito tabáquico en programas homólogos. Para los fumadores activos (o quienes recaen en el hábito mientras se ventilan) se podría considerar la alternativa de retirarlos del programa, pero debido a que el acceso a la terapia óptima para el abandono de este hábito no está disponible para todos los pacientes, puede ser más adecuado mantenerlos bajo VMNID y evitar así sus exacerbaciones. Actualmente, se recomienda no ingresar pacientes nuevos con FRGC que mantienen tabaquismo activo.

Destaca en la función pulmonar, 2 pacientes con espirometrías descritas como normales, las cuales corresponden a pacientes portadores de SHO, los cuales son jóvenes y con pulmón sano.

En salud mental, AVNIA realiza un tamizaje de depresión y ansiedad mediante el test de Goldberg (GHQ-12) a todos los ingresos, usando el formato abreviado de 12 preguntas auto administradas $^{4,19}$, según esta escala el $40 \%$ de los sujetos con FRGC posee sospecha de psicopatología (puntaje igual o mayor a 5). Por ello todos los pacientes que ingresan al programa de VMNID será referido al programa de salud mental de atención primaria de salud, para acceder a evaluación ${ }^{19}$. Revisado el sistema SIGGES sólo el 16,1\% tuvo una atención médica por Depresión en atención primaria, es probable, que exista una brecha en el acceso a salud mental de los sujetos con FRGC, lo que pudiese interferir, principalmente el manejo de la disnea y ansiedad. Se consideró la limitación funcional, reflejada en el requerimiento de órtesis por los sujetos con FRGC, la cual alcanzó un $23,6 \%$, esto asociado al hecho de que requieren oxígeno y ventilador, muestra la alta dependencia de ayudas externas para poder acceder a mejor calidad de vida.

Los pacientes con un ingreso mensual propio, menor a $\$ 264.000$ pesos chilenos (418 USD) fue el $92,1 \%$, reafirmando la vulnerabilidad de la muestra, los programas de VMNID deben contar con asistentes sociales que faciliten el acceso de los pacientes a los beneficios legales para mejorar sus condiciones de vida. La posibilidad de inserción laboral de sujetos con FRGC aptos es también un factor a considerar en el aspecto social. La precariedad de la vivienda y condiciones de vida de algunos usuarios de este programa (Tabla 1), requiere dedicación de los profesionales, respecto del cuidado de los pacientes, equipos e insumos.

Considerando el nivel educacional y limitaciones visuales de los pacientes con FRGC se sugiere que los programas de VMNID adapten sus protocolos de atención considerando estas variables en sus documentos de registro, para así entregar la información de la manera más comprensible (incluyendo al cuidador) y que ellos puedan entender su diagnóstico, pronóstico, farmacoterapia, orientación de emergencias respiratorias y el cuidado del equipamiento. El perfil del profesional que acude al domicilio debiese tener competencias sociales y humanitarias orientadas a este usuario particular.

En conclusión, los sujetos con FRGC de la red pública de salud en Chile son sujetos vulnerables, con factores de morbimortalidad y costosos para el sistema de salud, requieren para un manejo más efectivo, que los programas ventilatorios adopten además de un enfoque clínico, un enfoque socio-sanitario y estén insertos en la red de salud (principalmente en servicio social, salud cardiovascular y mental).

Las proyecciones son mejorar la gestión clínica de este programa, entregar información útil a grupos de trabajo relacionados y proporcionar a la autoridad sanitaria resultados para optimizar la entrega de recursos. Además, desarrollar el área de estudio de calidad de vida de los pacientes crónicos ventilados en Chile.

\section{Agradecimientos}

A todo el equipo de kinesiólogos y enfermeras del programa AVNIA que colaboraron en el cumplimiento del protocolo del trabajo durante todo el año 2016.

\section{Bibliografía}

1.- DÍAZ S, MAYORALAS S. La ventilación mecánica no invasiva moderna cumple 25 años. Arch Bronconeumol. 2013; 49: 475-9.

2.- MINISTERIO DE SALUD GOBIERNO DE CHILE. Estrategia Nacional de Salud Para el Cumplimiento de los Objetivos Sanitarios de la Década 2011-2020. Santiago; 2011. p.67. (Citado en agosto de 2017) Disponible en: http://www.ispch.cl/sites/default/files/ documento/2011/12/Metas2011-2020.pdf

3.- LLOYD-OWEN SJ, DONALDSON GC, AMBROSINO N, ESCARRABILL J, FARRÉ R, FAUROUX B, et al. Patterns of home mechanical ventilation use in Europe: results from the Eurovent survey. Eur Respir J, 25 (2005), pp. 1025-31 http://dx.doi.org/10.1183/0903193 6.05.00066704 Medline

4.- MINSAL, Chile. Norma Técnica: Programa de Asistencia Ventilatoria No Invasiva Adultos en Atención Primaria de Salud (AVNIA). Chile: División de Atención Primaria; Unidad de Salud Respiratoria; 2013. (Citado en agosto de 2017). Disponible en: https://respiratorio. 
minsal.cl/PDF/AVNI/Norma_AVIA_2013_snt_2_enero_2013.pdf.

5.- MINSAL, Chile, Nacional ENS. Encuesta nacional de salud. Pontificia Universidad Católica de Chile, Universidad Alberto Hurtado, Chile 2009-2010. (Citado en agosto de 2017) Disponible en: http://www.minsal.cl/ portal/url/item/bcb03d7bc28b64dfe040010165012d23. pdf

6.- LLOYD-OWEN SJ, DONALDSON GC, AMBROSINO $\mathrm{N}$, ESCARABILL J, FARRE R, FAUROUX B, et al. Patterns of home mechanical ventilation use in Europe: Results from the Eurovent survey. Eur Respir J. 2005; 25: 1025-31.

7.- WINDISCH W, FREIDEL K, SCHUCHER B, BAUMANN H, WIEBEL M, MATTHYS $H$, et al. The Severe Respiratory Insufficiency (SRI) Questionnaire A specific measure of health-related quality of life in patients receiving home mechanical ventilation. J Clin Epidemiol. 2003; 56: 752-9.

8.- LÓPEZ-CAMPOS L, FAILDE I, LEÓN JIMÉNEZ A, MASA JIMÉNEZ F, BARROT CORTÉS E, BENÍTEZ MOYA JM, et al. Calidad de vida relacionada con la salud de pacientes en programa de ventilación mecánica domiciliaria. La versión española del cuestionario SRI. Arch Bronconeumol. 2006; 42: 588-93.

9.- GHOSH D, RZEHAK P, ELLIOTT MW, WINDISCH W. Validation of the English severe respiratory insufficiency questionnaire. Eur Respir J. 2012; 40: 408-15.

10.- ASSOCIATION NATIONALE POUR LE TRAITEMENT A DOMICILE DES INSUFFISANTS RESPIRATOIRES (Francia) octubre de 2017, disponible en: http://www.antadir.com/fr/la-federation.

11.- BRITISH THORACIC SOCIETY, Guideline: The Use of Non-Invasive Ventilation in the management of patients with chronic obstructive pulmonary disease admitted to hospital with acute type II respiratory failure (With particular reference to Bilevel positive pressure ventilation), 2008. Disponible en: https://www.britthoracic.org.uk/document-library/clinical-information/ niv/niv-guidelines/btsrcpics-guideline-on-niv-in-copd/
12.- FERRERO G. Consenso Chileno de Ventilación Mecánica No Invasiva, XII.- Modos Ventilatorios en VNI, Rev Chil Enferm Respir 2008; 24: 240-50.

13.- JALILIE, A MAQUILÓN C, TOMICIC V, LIRA P. Consenso Chileno de Ventilación Mecánica No Invasiva, X. Complicaciones y estándares de cuidado de la VNI. Rev Chil Enferm Respir 2008; 24: 233-6.

14.- FONDO NACIONAL DE SALUD (FONASA). (Citado en agosto de 2017). Disponible en: https://www.fonasa. $\mathrm{cl} /$ sites/fonasa/beneficiarios/coberturas/auge/patologias.

15.- GOBIERNO DE CHILE, FONDO NACIONAL DE SALUD (FONASA), Sistema de Gestión de Garantías Explícitas en Salud (SIGGES), años 2016-2017. (Citado en agosto de 2017). Disponible en: http://www.sigges.cl.

16.- GOBIERNO DE CHILE, FONDO NACIONAL DE SALUD (FONASA), Departamento de Planificación Institucional, Comunicaciones y Marketing. Cuenta Pública Participativa, año 2016. (Citado en agosto de 2017). Disponible en: http://fonasaweb.fonasa.cl/portal_fonasa/stat/CPP2016/CuentaPublica_gestion2016. pdf

17.- ANTOLINI M, OLAVE C, ALARCÓN G, ANDRADE M, VERA R, MAQUILÓN C, et al. Niveles de dióxido de carbono por medición de gases arteriales y por medición transcutánea en pacientes adultos en VMNI. Rev Chil Enferm Respir 2015; 31 (Supl): 531 (Resumen CL-37).

18.- WINDISCH W, FREIDEL K. Evaluation of Healthrelated quality of life using the MOS 36 Item Short from Health Status survey in patients receiving noninvasive positive pressure ventilation Intensive Care Med 2003; 29: 615-62.

19.- MINISTERIO DE SALUD, GOBIERNO DE CHILE SUBSECRETARIA DE SALUD PÚBLICA. División de Prevención y Control de Enfermedades. Guía Clínica AUGE: Depresión en personas de 15 años y más. Serie Guías Clínicas MINSAL, 2013. (Citado en agosto de 2017). p. 38. Disponible en: http://www.minsal.cl/ portal/url/item/7222754637c08646e04001011 f014e64. pdf.

\footnotetext{
Correspondencia a:

Dr. César Maquilón O.

Médico especialista broncopulmonar, Oficina de Coordinación del Servicio Médico Quirúrgico.

Clínica Dávila, Avda. Recoleta 464, Edificio “D”, 5 ○

piso sur, Comuna de Recoleta, Santiago, Chile.

Código Postal: 8431657

Email: cmaquilon87@hotmail.com
} 